Original breve

ISSN 2519-0652 CASUS.2017;2(2):100-105.

\title{
Factores asociados al conocimiento sobre el examen de Papanicolaou en mujeres peruanas
}

\section{María Arévalo-Ochoa ${ }^{1}$}

Rosa Arévalo-Ochoa ${ }^{1}$

RESUMEN: El objetivo del estudio fue determinar qué factores sociodemográficos se encuentran asociados al nivel de conocimiento sobre el examen del Papanicolaou. La muestra fue de 130 mujeres entre los 20 a 40 años que han sido atendidas en centros de la Red de Salud Lima Norte V. De las 130 mujeres un $70 \%$ tuvo un nivel alto de conocimiento sobre el Papanicolaou. Comenzar la actividad sexual antes de los 20 años y haberse realizado el examen de Papanicolaou se asociaron con un alto nivel de conocimiento. Se sugiere que el personal de enfermería ejecute campañas de salud preventiva promocional respecto al cáncer de cuello uterino, con el fin de aumentar el conocimiento de la población en general, teniendo en cuenta que el inicio temprano de la actividad sexual es un factor de riesgo para contraer ITS.

PALABRAS CLAVE: Prueba de Papanicolaou; Conocimiento; Salud de la mujer.

Citar como: Arévalo M, Arévalo R. Factores asociados al conocimiento sobre el examen de Papanicolaou. CASUS. 2017; 2(2):100-105. 


\section{INTRODUCCIÓN}

El cáncer es una enfermedad que puede golpear cualquier órgano del cuerpo, ya sea en el hombre o la mujer afectando a la salud. A nivel mundial el cáncer es una de las principales causas de muerte. En el 2012 causó 1.3 millones de muertes y un $47 \%$ ocurrió en América Latina y el Caribe (1). Cabe recalcar que en Latinoamérica ocupa el tercer lugar como causa de muerte (2). Respecto a los tipos de cáncer el de cuello uterino se coloca en el segundo lugar entre las mujeres que viven en regiones menos desarrolladas (3). Asimismo, uno de sus factores de riesgo es el virus de papiloma humano (VPH) que es transmitido por actividad sexual $(1,4,5)$.

En el Perú entre los diagnósticos de cáncer el de cérvix ocupó el primer lugar desde el 2000 hasta el 2014. En el 2012 hubo 4636 casos y 1715 muertes por cáncer de cuello uterino. La incidencia estandarizada por edad es de 32.7 años. Los lugares en donde hay mayor frecuencia de cáncer es en Loreto (29.4\%), Ucayali (28.6\%), Madre de Dios (28.5\%) y Moquegua (28.4\%) y la región que cuadruplica la tasa ajustada de mortalidad por cáncer de cuello uterino es Lima (6). Gran parte de estos eventos se pudieran prevenir con una prueba de detección adecuada como es el examen de Papanicolaou (PAP) (6).

El PAP se usa para detectar el cáncer del cuello uterino. El mismo puede ser realizado por el personal capacitado y es una prueba sencilla. Se realiza mediante el raspado del tejido, se coloca en el fijador y se procede a la coloración especial para luego ser examinada en el microscopio $(7,8)$. Otro procedimiento es la inspección visual mediante la colocación de ácido acético al 5\% en la cérvix y el tejido anormal toma un color blanco (6). El examen se realiza una vez al año desde que se inicia la primera actividad sexual (6).

En un estudio realizado en mujeres en edad fértil sobre conocimiento del examen del Papanicolaou aquellas que iniciaron su actividad sexual entre $15 \mathrm{y}$ 20 años tuvieron un mayor conocimiento sobre la prueba (9-11). Mientras que otro estudio reporta que las mujeres que conocen el beneficio del PAP se han realizado alguna vez este examen (12).

Teniendo en cuenta la problemática que causa el cáncer de cérvix, las necesidades de mejorar las estrategias de prevención temprana y los escasos estudios sobre factores sociodemográficos relacionados al nivel de conocimiento sobre el examen de Papanicolaou, el presente estudio tuvo como objetivo determinar factores que se asocian al nivel de conocimientos sobre el examen del Papanicolaou.

\section{MATERIALES Y MÉTODOS}

El estudio fue descriptivo-correlacional de diseño transversal. La muestra estuvo conformada por 130 mujeres de 20 a 40 años de edad que fueron atendidas en los centros de salud de la Red Lima Norte V. El muestreo fue no probabilístico. Se incluyeron solo a mujeres en edad fértil usuarias que acudían a los centros de salud y que se hayan realizado o no el examen de PAP. Se excluyeron aquellos que no habían iniciado su actividad sexual.

La variable principal conocimiento sobre el examen del Papanicolaou se midió a través de un cuestionario de 10 preguntas, validado a nivel nacional (12) que indagaba en la definición, frecuencia de la realización, requisitos para tomar la muestra, factores de riesgo, personas dirigidas a realizarse el examen entre otros temas. La variable fue categorizada en alto, regular y bajo.

Respecto a los factores sociodemográficos se consideraron: la edad, variable numérica; estado civil variable categórica (soltera, casada y conviviente); grado de instrucción variable categórica (primaria, secundaria, superior no universitario, superior universitario); número de hijos variable numérica; edad de inicio de la actividad sexual, variable categórica (menor de 15, 15 a 20 y mayor de 20 años); número de parejas sexuales, variable categórica (solo una pareja sexual, de 2 a 3 parejas sexuales y mayor de 3 parejas sexuales); uso de método anticonceptivo variable categórica (píldora, inyectable, condón y ninguno).

Para el análisis de datos se usó el programa IBM SPSS versión 24.0. En el análisis descriptivo se emplearon los porcentajes para las variables categóricas y la media para las variables numéricas. Para el análisis bivariado se emplearon las pruebas de Chi-cuadrado y ANOVA con una significancia menor igual 0.05. El estudio contó con la aprobación de un Comité de Ética de Investigación. Igualmente se respetó la confidencialidad y el anonimato de las personas encuestadas. 


\section{RESULTADOS}

De las 130 mujeres la edad media fue de 28.65 años y la media del números de hijos fue de 1.33. El 41.5\% de las mujeres eran solteras, el $46.9 \%$ reportó la secundaria como un nivel educativo, el $27.7 \%$ usó un inyectable como método anticonceptivo. La mayor parte de las mujeres iniciaron su actividad sexual entre los 15 y los 20 años de edad (71.5\%), tuvieron de dos a tres parejas sexuales $(52.3 \%)$, se realizaron alguna vez el Papanicolaou (70\%) y tenía un nivel alto de conocimiento sobre PAP (70\%) (Ver tabla 1).

Tabla 1. Descripción de las características de la muestra.

\begin{tabular}{l}
\hline \\
Número de hijos (media) \\
Edad (media) \\
Estado civil \\
Soltera \\
Casada \\
Conviviente \\
Grado de instrucción \\
Primaria \\
Secundaria \\
Superior \\
Edad de inicio de la actividad sexual \\
menor de 15 \\
de 15 a 20 \\
mayor de 20
\end{tabular}

Número de parejas sexuales
Solo uno
de 2 a 3
mayor a 3
Método anticonceptivo
Condón
Inyectable
Píldora
Ninguno
Otros
Realización del Papanicolaou
Si
No
Nivel de conocimiento sobre Papanicolaou
Alto
Regular
Bajo

36.2

52.3

11.5

27.7

26.9

4.6

70

30
Iniciar la actividad sexual entre los 15 y los 20 años se asoció significativamente con el alto nivel de conocimiento sobre el examen de PAP en las mujeres (69.9\%). Asimismo, el haber realizado en alguna ocasión el examen de Papanicolaou se relacionó con un alto nivel de conocimiento $(79.1 \%)$. El resto de variables no resultó estadísticamente significativo (ver tabla 2).

Tabla 2. Asociación descriptiva entre los factores sociodemográficos y el nivel de conocimiento sobre el Papanicolaou.

\begin{tabular}{|c|c|c|c|c|}
\hline & \multicolumn{4}{|c|}{ Nivel de conocimiento } \\
\hline & $\begin{array}{l}\text { Alto } \\
\mathrm{n}(\%)\end{array}$ & $\begin{array}{c}\text { Regular } \\
\mathrm{n}(\%)\end{array}$ & $\begin{array}{l}\text { Bajo } \\
\mathrm{n}(\%) \mathrm{p}\end{array}$ & $p$-value \\
\hline $\mathrm{N}^{\circ}$ de hijos (media & a) 1.24 & 1.57 & 0.90 & 0.36 \\
\hline Edad (media) & 28.9 & 28.2 & 27.2 & 0.25 \\
\hline \multicolumn{5}{|l|}{ Estado civil } \\
\hline Soltera & $37(68.5)$ & $12(22.2)$ & $5(9.3)$ & \\
\hline Casada & $17(70.8)$ & $4(16.7)$ & $3(12.5)$ & 0.381 \\
\hline Conviviente & $37(71.2)$ & $14(26.9)$ & $1(1.9)$ & \\
\hline \multicolumn{5}{|c|}{ Grado de instrucción } \\
\hline Primaria & $6(66.7)$ & $2(22.2)$ & 1(11.1) & \\
\hline Secundaria & $37(60.7)$ & 18(29.5) & $6(9.8)$ & 0.208 \\
\hline Superior & $48(80.0)$ & 10(16.7) & $2(3.3)$ & \\
\hline \multicolumn{5}{|c|}{$\begin{array}{l}\text { Edad de inicio de la } \\
\text { actividad sexual }\end{array}$} \\
\hline Menor de 15 & $3(50.0)$ & 1(16.7) & $2(33.3)$ & \\
\hline Entre 15 a 20 & $65(69.9)$ & $25(26.9)$ & $3(3.2)$ & 0.017 \\
\hline Mayor de 20 & $23(74.2)$ & $4(12.9)$ & $4(12.9)$ & \\
\hline
\end{tabular}

\section{Número de parejas sexuales}

$\begin{array}{lllll}\text { Solo uno } & 31(66.0) & 11(23.4) & 5(10.6) & \\ \text { De 2 a 3 } & 49(72.1) & 15(22.1) & 4(5.9) & 0.667 \\ \text { Mayor a } 4 & 11(73.3) & 4(26.7) & 0(0.0) & \end{array}$

24.6

16.2

\section{Método anticonceptivo}

Condón

22(68.8) $8(25.0) \quad 2(6.2)$

Inyectable

$26(72.2) \quad 8(22.2) \quad 2(5.6)$

Píldora $\begin{array}{llll}15(71.4) & 4(19.0) & 2(9.5) & 0.994\end{array}$

Ninguno

24(68.6) $8(22.9) \quad 3(8.6)$

\section{Mujeres que se realizan}

el Papanicolaou

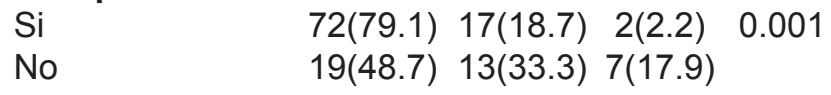




\section{DISCUSIÓN}

Los resultados muestran que un $70 \%$ de las encuestadas tuvo un nivel alto de conocimiento sobre Papanicolaou. Asimismo, el iniciar la actividad sexual antes de los 20 años y realizarse con anterioridad el examen de Papanicolaou se asociaron con un alto nivel de conocimiento sobre este examen.

En el estudio uno de los factores asociados al alto nivel de conocimiento sobre el PAP fue el haber iniciado la actividad sexual entre los 15 y los 20 años. En estudios similares se reporta este resultado $(9,10)$. Actualmente en el país hay un mayor y fácil acceso a la información sobre infecciones de transmisión sexual (ITS) a través de los medios de comunicación.

De la misma manera, en diversos establecimientos de salud y otras instituciones incentivan a la población juvenil al uso de métodos para detectar las ITS como es el caso del examen de Papanicolaou. Por otro lado, se ha relevado un mayor interés en la población joven que ha iniciado su actividad sexual con el fin de prevenir estas infecciones $(10,13)$.

Otro hallazgo importante fue que realizarse el examen del Papanicolaou se asoció con un alto nivel de conocimiento en las encuestadas. Otro estudio detalla que las mujeres que se realizan el examen de Papanicolaou conocen sobre esta prueba (12). Las mujeres que se realizan este examen generalmente reciben información precisa sobre sus beneficios por parte del personal de salud que les atiende, lo que influiría positivamente en la decisión de realizarlo (12).
Entre las limitaciones del estudio se señala que no se pudo obtener una muestra amplia debido al rechazo de las participantes a ofrecer datos personales sensibles que recopilaba la encuesta. Asimismo entre las limitaciones del diseño se encuentra el sesgo de memoria y temporalidad. Se recomienda para futuros estudios considerar muestras más amplias, incluir variables como prácticas y actitudes sobre el examen de Papanicolaou y realizar comparaciones entre centros, hospitales u otros establecimientos de salud.

\section{CONCLUSIONES}

Se asociaron con un alto nivel de conocimiento de la prueba el inicio temprano de la actividad sexual y la realización previa del examen de Papanicolaou. Se sugiere que el personal de enfermería ejecute campañas de salud preventivo-promocionales respecto al cáncer de cuello uterino en centros educativos, establecimientos de salud entre otras instituciones con el fin de aumentar el conocimiento de la población en general, teniendo en cuenta que el inicio temprano de la actividad sexual es un factor de riesgo para contraer ITS.

\section{REFERENCIAS BIBLIOGRÁFICAS}

1. Organización Panamericana de la Salud. Análisis situacional del cáncer de cuello uterino en América Latina y el Caribe [internet]. América Latina y el Caribe: OPS; 2016. [citado el 9 de agosto de 2017] Disponible en: http://www.paho.org/hq/index.php?o ption $=$ com_content $\&$ view $=$ article $\&$ i $\mathrm{d}=292$ \&Itemid $=3904 \&$ lang $=$ es.

2. Instituto Nacional de Enfermedades Neoplásicas. Datos epidemiológicos [internet]. Perú: INEN; 2017 [citado, 9 de agosto del 2017]. Disponible en: http://www.inen.sld.pe/portal/estadis ticas/datos-epidemiologicos.html.

3. Organización Mundial para la Salud. Papiloma virus humano (PVH) y cáncer cérvico uterino [internet]. Ginebra: OMS; 2015 [citado el 9 de agosto de 2017]. Disponible en: http://www.who.int/mediacentre/facts heets/fs380/es/

4. Organización Mundial de la Salud. Temas de salud [internet]. Ginebra: OMS; 2017 [citado de 9 de agosto de 20016 ]. D i s p o n i b 1 e en: http://www.who.int/topics/cancer/ es/.

5. Soto OD. VPH y cáncer de cérvix. Revista Médica de Costa Rica y C e n t r o a m é r i c a. 2013 ; 70(607):417-421.

6. ISO 690 Ministerio de Salud, editores. Guía de práctica clínica para la prevención y manejo de cáncer de cuello uterino: guía técnica [internet]. Lima: Resolución Ministerial; 2016 [citado el 9 de agosto de 2017]. Disponible en:

//ftp2.minsa.gob.pe/normaslegales/2 016/RM-N_1013-2016-MINSA.pdf.

7. Serva B y Soto M. Nivel de conocimientos y actitudes del tamizaje de Papanicolaou en mujeres que acuden al C.S de Chupaca de Enero - Marzo 2016 [tesis de licenciatura]. Huancayo: Universidad Peruana del Centro; 2016

8. Pérez G, Silva P, Palma R. Manual de Procedimientos para Papanicolaou en Hospital Regional Rancagua [internet]. Chile: Hospital Regional de Rancagua; 2015 (2 de Marzo de 2015). Disponible en:

portal.mspas.gob/files/Descargas/Pro tecciondelaSalud/componentecancer 
/MANUAL\%20PARA\%20TAMIZA JE\%20DEL\%20CANCER\%20CER VICO\%20UTERINO

9. American Cancer Society. Factores de riesgo del cáncer uterino [internet]. EE.UU:CANCER.ORG; 2017. [citado el 9 de agosto de 2017]. Disponible en:

https://www.cancer.org/es/cancer/ca ncer-de-cuello-uterino/causas-riesgo s-prevencion/factores-de-riesgo.html

10. Motta A, Keogh S, Prada E, Nuñez A, Konda K, Stilman M, et al. De la normativa a la práctica: la política de la educación sexual y su implementación en el Perú [internet]. New York: Guttmacher institute; 2017 [citado el 10 de agosto de 2017]. Disponible en:

https://www.guttmacher.org/sites/def ault/files/report_pdf/de-la-normativa -a-la-practica-educacion-sexual-peru .pdf.
11. Zamberline N, Thouyaret L, Arrosi S. Lo que piensan las mujeres: conocimientos y percepciones sobre el cáncer del cuello uterino y la realización del PAP, Buenos Aires: Ministerio de Salud de la Nación. Programa Nacional de Prevención de Cáncer Cérvico Uterino, 2013, http://www.msal.gob.ar/images/stori es/bes/graficos/0000000286cnt-32-L o_que_piensan_las_mujeres.pdf.

12. Carrasco JR, Valera LM. Relación entre el nivel de Conocimiento, Actitudes y Practicas sobre el examen de Papanicolaou en mujeres en edad fértil sexualmente activas de la urbanización 'Las Palmeras' en el distrito de Morales, Julio Diciembre 2011 [Tesis de licenciatura]. Tarapoto: Universidad Nacional de San Martin; 2011.
13. Yuri Paulin Holguin, Luis Alfonzo Mendoza, Claudia Marcela Esquivel, et al. Factores Asociados al Inicio de la Vida Sexual en Adolescentes de Tulua, Colombia. Revista Chilena Ginecología Obstetricia. 2013;78(3), 209-219.

14. Gutiérrez C, Romaní F, Ramos J. Factores asociados con el conocimiento y tamizaje para cáncer de cuello uterino (examen de Papanicolaou) en mujeres peruanas en edad fértil. Análisis del periodo 1996-2008. Revista Peruana de Epidemiología. 2010;14(1). 


\title{
Factors associated with the knowledge about the Papanicolaou examination in Peruvian women
}

\begin{abstract}
The aim of this study was to determine which sociodemographic factors are associated with the level of knowledge about the Pap smear. The sample consisted of 130 women between the ages of 20 and 40 years who had been treated in centers of the Health Network North Lima V. Of the 130 women $70 \%$ have a high level of knowledge about the Papanicolaou. Beginning sexual activity before the age of 20 and having undergone the Pap test were associated with a high level of knowledge. It is suggested that nurses carry out promotional preventive health campaigns regarding cervical cancer. In order to increase awareness of the general population, taking into account that the early onset of sexual activity is a risk factor for contracting STIs.
\end{abstract}

KEY WORDS: Papanicolaou test; Knowledge; Information society; Women's Health. 